\title{
Electron affinity and surface states of GaN $m$-plane facets: Implication for electronic self-passivation
}

\author{
V. Portz, ${ }^{1}$ M. Schnedler, ${ }^{1}$ H. Eisele, ${ }^{2}$ R. E. Dunin-Borkowski, ${ }^{1,3}$ and Ph. Ebert ${ }^{1, *}$ \\ ${ }^{1}$ Peter Grünberg Institut, Forschungszentrum Jülich GmbH, 52425 Jülich, Germany \\ ${ }^{2}$ Technische Universität Berlin, Institut für Festkörperphysik, Hardenbergstr. 36, 10623 Berlin, Germany \\ ${ }^{3}$ Ernst Ruska-Centrum, Forschungszentrum Jülich GmbH, 52425 Jülich, Germany
}

(Received 27 November 2017; revised manuscript received 15 February 2018; published 20 March 2018)

\begin{abstract}
The electron affinity and surface states are of utmost importance for designing the potential landscape within (heterojunction) nanowires and hence for tuning conductivity and carrier lifetimes. Therefore, we determined for stoichiometric nonpolar $\mathrm{GaN}(10 \overline{1} 0) m$-plane facets, i.e., the dominating sidewalls of $\mathrm{GaN}$ nanowires, the electron affinity to $4.06 \pm 0.07 \mathrm{eV}$ and the energy of the empty Ga-derived surface state in the band gap to $0.99 \pm 0.08 \mathrm{eV}$ below the conduction band minimum using scanning tunneling spectroscopy. These values imply that the potential landscape within GaN nanowires is defined by a surface state-induced Fermi-level pinning, creating an upward band bending at the sidewall facets, which provides an electronic passivation.
\end{abstract}

DOI: 10.1103/PhysRevB.97.115433

\section{INTRODUCTION}

Group III-nitride semiconductor nanowires (NWs) are highly promising building blocks for energy harvesting and optoelectronic applications due to the exceptional ability of enhanced strain relaxation. Hence, NW heterojunctions can be grown defect-free for a significantly wider range of material combinations as compared to planar structures. However, NWs exhibit a large surface-to-volume ratio [1,2] and thus their electronic properties are significantly affected by surface effects [3-5]. The dominating facets of wurtzite structure NWs are the $m$-plane sidewall facets, whose properties are of prime interest. Among the surface-related properties, the electron affinity and the energetic positions of surface states at these $m$ plane sidewall facets are of special interest for understanding the electronic properties of NWs and their heterojunctions.

The electron affinity $\chi$ of a semiconductor is defined as the energy required to excite an electron from the conduction band minimum $\left(E_{\mathrm{C}}\right)$ to the vacuum level right outside the semiconductor $\left(E_{\mathrm{vac}}\right)$, i.e., $\chi=E_{\mathrm{vac}}-E_{\mathrm{C}}$ [6,7]. At first glance, the definition appears straightforward and the electron affinity is frequently considered to be a material constant. However, e.g., for GaN, no commonly accepted value for the electron affinity exists. A literature survey reveals a broad range of experimentally and theoretically obtained values spanning from $2.1 \mathrm{eV}$ to $4.26 \mathrm{eV}$ [8-19]. It is important to note that all experimental values were probed at polar GaN surfaces. To understand the wide spread of values, we recall that $\chi$ consists of two components: (i) a material component connected to the electronic density of states of the material and (ii) a surface component connected to dipoles, polarizations, and atomic rearrangements, as well as adsorption or other phenomena that affect the potential in the vacuum [20]. Unfortunately, experimentally, these two components could not be separated thus far and, as a matter of fact, the polarization as well as

\footnotetext{
*p.ebert@fz-juelich.de
}

the almost omnipresent Ga adlayers [21] modify the electron affinity at polar GaN surfaces. Hence, for achieving a separation of the material and surface component of the electron affinity, one needs a surface free of dipoles, polarization, atomic rearrangements, adsorption, or adlayers. This can be realized on nonpolar, stoichiometric GaN $m$-plane surfaces, which do not have any of the above-mentioned problems and promise to access the material component of the electron affinity only.

Furthermore, for probing the material component of $\chi$ on nonpolar GaN $m$-plane surfaces, we need to consider possible band bending near the surface and thus the presence of intrinsic surface states. However, an exceptionally broad energy range of the empty Ga-derived dangling bond surface state has been reported [22-31]: Even the most recent measurements cover a rather wide energy range between $0.5 \mathrm{eV}$ and $1.1 \mathrm{eV}$ below the conduction band minimum [24,25,28-31], pinning the Fermi energy $\left(E_{\mathrm{F}}\right)$ in the fundamental band gap [32]. The origin of the widely varying energy determinations is unclear at present, but is relevant to the electron affinity.

Therefore, we use scanning tunneling spectra acquired on clean and stoichiometric nonpolar GaN(1010) $m$-plane surfaces, freshly cleaved in UHV, to extract the material component of the electron affinity and the energetic position of the unoccupied Ga-derived surface state. We illustrate the impact of these surface properties on the charge separation and recombination, demonstrating the electronic self-passivation of GaN NWs.

\section{EXPERIMENT}

For our investigations, 500-nm-thick $S i$-doped GaN epitaxial layers ( $n$-type carrier concentration of $3 \times 10^{18} \mathrm{~cm}^{-3}$ calibrated by $\mathrm{C}-\mathrm{V}$ profiling and secondary ion mass spectroscopy) grown on free-standing $c$-plane $\mathrm{GaN}$ pseudosubstrates by metal organic vapor phase epitaxy were cleaved in ultrahigh vacuum $\left(p \approx 1 \times 10^{-8} \mathrm{~Pa}\right)$. No other impurities were detected. The freshly cleaved (10) 10$)$ surfaces were investigated in situ 




FIG. 1. Scanning tunneling spectrum of GaN acquired at constant tip-sample separation fixed at a set point of $-3.6 \mathrm{~V}$ and $150 \mathrm{pA}$ (black dots). The blue, red, and green lines show simulations of the $I(V)$ spectrum assuming different electron affinities. Spectra simulated with smaller $\chi$ values exhibit steeper slopes and hence significantly larger tunnel currents. The best fit to the tunnel spectrum near the onset voltages (see text) was achieved by assuming an electron affinity of $3.6 \mathrm{eV}$, shown as red line. For the simulation, the tip-sample separation and the energy of the empty surface state are used as fitting parameters too. The inset shows a 500-nm-wide GaN buffer used for the acquisition of tunneling spectra.

by cross-sectional scanning tunneling microscopy (STM) and spectroscopy (STS) using electrochemically etched tungsten (W) tips.

The cleavage surfaces are atomically flat, with low step density and defect concentration (see inset in Fig. 1). Tunneling spectra were experimentally obtained on various cleavage surfaces with various tips and fitted using SEMITIP, version 6, UniInt2 [33]. These simulations of the tunnel current take into account the Fermi-level pinning by intrinsic surface states, the tip-induced band bending, and the screening by free carriers.

\section{RESULTS AND DISCUSSION}

\section{A. Electron affinity}

An example of an experimentally obtained $I(V)$ tunnel spectrum is shown by the black dots in Fig. 1. The spectrum was acquired on $\mathrm{GaN}$ using a set point of $-3.6 \mathrm{~V}$ and $150 \mathrm{pA}$. Note, we chose tunnel current set points small enough such that the tip did not modify the surface. The red, green, and blue lines represent fits to the tunnel spectrum assuming different electron affinities. The electron affinity has a strong influence on the tunnel current and affects the onset voltages as well as the slope of the tunnel spectra. Spectra derived with smaller $\chi$ values exhibit steeper slopes and hence significantly larger tunnel currents. Additionally, the onset of the current moves to higher absolute voltages with smaller $\chi$. Note, to obtain the best fits to the experimental tunnel spectra, we took into account that the parabolic band approximation in the simulation leads to the highest accuracy near the band edges. Hence the tunnel current and its slope near the of onset voltages were optimized. Furthermore, the onset at positive voltages is influenced by


FIG. 2. Electron affinity values $\chi_{\mathrm{GaN}}$ extracted from simulations fitted to scanning tunneling spectra obtained experimentally at different set points with different $\mathrm{W}$ tips. The electron affinity is plotted as gray dots as a function of (a) the set voltage and (b) the fitted energy of the unoccupied surface state. The weighted mean $\chi_{\mathrm{GaN}}=$ $4.06 \pm 0.07 \mathrm{eV}$ with standard error is shown as a red (a) line and (b) circle. The electron affinity and the set voltage are not correlated. The Ga-derived empty surface state is located $0.99 \pm 0.08 \mathrm{eV}$ below the conduction band minimum.

the position of the empty Ga-derived surface state below the conduction band edge $\left(E_{\mathrm{C}}-E_{\mathrm{SS}}\right)[28,32]$. The best fit to the illustrated, experimentally obtained tunnel spectrum is shown as a red line in Fig. 1 and was achieved by using an electron affinity of $3.6 \mathrm{eV}$, a tip-sample separation of $1.26 \mathrm{~nm}$, and a surface state $1.09 \mathrm{eV}$ below $E_{\mathrm{C}}$.

In this way, simulations were fitted to various experimentally obtained tunneling spectra acquired at different set points and with different tips. From each simulation, the electron affinity and the energy of the surface state are extracted. The gray dots in Figs. 2(a) and 2(b) show the obtained electron affinity values as a function of the set voltage and the energy of the surface state relative to the conduction band, respectively. The diagrams reveal that the obtained electron affinity values are not correlated to the applied voltage and independent of the energy of the surface state.

The values of $\chi$ range from $2.6 \mathrm{eV}$ to $4.85 \mathrm{eV}$ with a weighted mean of $\chi_{\mathrm{GaN}}=4.06 \pm 0.07 \mathrm{eV}$ (red line and red symbol in Figs. 2(a) and 2(b), respectively). To understand the 
scattering of electron affinity values, we recall that, to a large extent, the onset voltages and slope of the tunnel spectra are determined by the shape of the tunnel barrier and the band bending. The latter two are equally affected by the electron affinity of the semiconductor and the work function of the tip as well as by the tip-sample separation [34]. First, we address the work function of the tip: The most commonly used value for the work function of tungsten tips is $4.5 \mathrm{eV}$. However, if impurity atoms adsorb at the tip apex or if the tip apex exhibits different crystal orientations, the work function of the tip changes. It was shown that the work function of W tips can vary between 3.9 and $5.5 \mathrm{eV}$ [35]. In addition, the shape of the tip influences the tunnel spectra, although to a smaller extent. Despite the same manufacturing process, no tip has an identical shape and both the shape and apex radius of a tip may change during measurement. Since it is not possible to obtain the electron affinity of the sample and the tip's work function independently from STS, we assume an average tip work function of $4.5 \mathrm{eV}$. If we assume a larger (smaller) work function for the tip, the electron affinity would roughly change by an equal value. Hence, for an uncertainty of the tip work function of $0.5 \mathrm{eV}$, an additional error of $0.5 \mathrm{eV}$ should be added to the measured electron affinity value. Since the tip-sample separation as fit parameter has a marginal impact on the onset voltages and slope [36], a reasonably realistic value for the electron affinity of the contamination-free and stoichiometric $m$-plane GaN surface is obtained.

The determined value of $\chi_{\mathrm{GaN}}=4.06 \pm 0.07 \mathrm{eV}$ ranges at the upper end of the spectrum of literature values. As outlined in the introduction, the electron affinity at the nonpolar surface is primarily given by the material component, whereas polarization, nonstoichiometry, Ga adlayers, etc. arising from annealing, decomposition, or adsorption effects at (0001) or $(000 \overline{1})$ surfaces lower the electron affinity. This interpretation is supported by electron affinities differing by $0.5 \mathrm{eV}$ for $\mathrm{GaN}(0001)$ and $(000 \overline{1})$ surfaces [15,37], suggesting relevant effects of the opposite spontaneous polarization. On our nonpolar surface, no polarization perpendicular to the surface is present. Finally, for a nonpolar, relaxed $m$-plane GaN surface, an electron affinity between 3.4 and $4.0 \mathrm{eV}$ was calculated, fluctuating systematically with the calculational method used [38]. This range agrees rather well with our experimental value.

\section{B. Energy of Ga-derived empty surface state}

In addition to the electron affinity, the energy of the Gaderived empty surface state relative to the conduction band minimum can be extracted from the simulation of the tunnel current and is found to lie $0.99 \pm 0.08 \mathrm{eV}$ below the conduction band minimum [Fig. 2(b)].

In recent literature, varying energy positions of the empty surface state on the $m$-plane GaN surface state were reported. These differences can be attributed to the way the various measurement methods probe the density of states (DOS). For this, we recall the band structure and the DOS of the relaxed $\mathrm{GaN}(10 \overline{1} 0)$ surface as calculated by Lymperakis et al. in Figs. 3(a) and 3(b), respectively [28]. Gray areas mark the projected bulk band structure and DOS. The empty Ga-derived $\left(S_{\mathrm{Ga}}\right)$ and occupied $\mathrm{N}$-derived $\left(S_{\mathrm{N}}\right)$ surface state are shown in red. In the DOS, the peak of the Ga-derived surface state

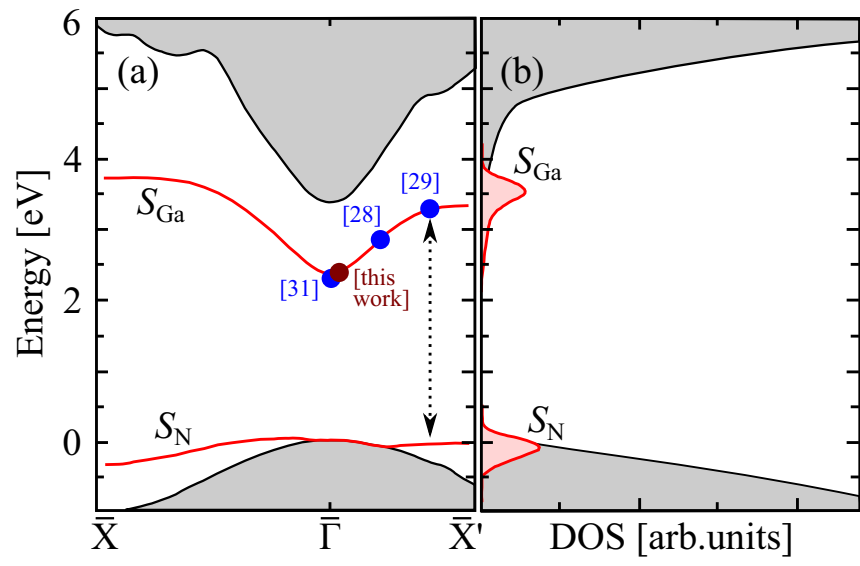

FIG. 3. GaN(1010) (a) band structure and (b) corresponding DOS as calculated in Ref. [28]. Gray areas mark the projected bulk band structure and the related DOS. The empty Ga-derived and the filled $\mathrm{N}$-derived surface states are shown in red. The blue and dark red symbols mark experimentally obtained energies of the empty surface state projected for illustration purposes onto the calculated $\bar{\Gamma}-\overline{\mathrm{X}}^{\prime}$ branch of the surface band structure. The distribution of the measured energies on the band structure suggests that the different experiments probe different parts of the DOS.

overlaps with the onset of the bulk conduction band. However, the surface state exhibits a long tail with a low DOS extending into the fundamental band gap. The blue and dark red symbols mark experimentally obtained energies of the surface state projected for illustration purposes onto the calculated $\bar{\Gamma}-\overline{\mathrm{X}}^{\prime}$ branch of the surface band structure. The distribution of the measured energies on the band structure suggests that the different experiments probe different parts of the DOS.

First, the optical surface adsorption peak of $3.3 \mathrm{eV}$ measured by reflection anisotropy spectroscopy (RAS) of the $\mathrm{GaN}(10 \overline{1} 0)$ surface by Himmerlich et al. [29] is based on electron transitions, and thus corresponds to the surface band gap, where the overlap of empty and filled surface DOS is maximized. Figures 3(a) and 3(b) show that the flat parts of the surface dispersion, i.e., corresponding to a larger DOS, are located near the edges of the surface Brillouin zone. The surface band gap is calculated to be $4.1 \mathrm{eV}$ and $3.3 \mathrm{eV}$ at the $\overline{\mathrm{X}}$ and $\overline{\mathrm{X}}^{\prime}$ points, respectively. Hence, RAS is most likely probing the $\overline{\mathrm{X}}^{\prime}$ point. Taking into account the calculated [28] and recently measured [31] dispersion, the filled surface state is at the $\overline{\mathrm{X}}^{\prime}$ point $0.1-0.2 \mathrm{eV}$ below the valence band maximum $\left(E_{\mathrm{V}}\right)$. Hence, the empty surface state is $0.2-0.3 \mathrm{eV}$ below $E_{\mathrm{C}}$ at the $\overline{\mathrm{X}}^{\prime}$ point.

Second, in contrast, normalized conductivity $(\mathrm{d} I / \mathrm{d} V) /(\overline{I / V})$ measurements in tunneling spectroscopy of Lymperakis et al. [28] probe the density of states, which extends further into the vacuum. Since the decay of the DOS is convoluted by the wave vector, STS probes the states with smallest possible wave vectors, but still largest DOS. Hence, the surface state is found deeper in the band gap $\left(E_{\mathrm{C}}-0.6 \mathrm{eV}\right)$ as compared to RAS.

Third, the Fermi energy is pinned at the minimum of the empty surface state located at the $\bar{\Gamma}$ point. This pinning has been measured by probing $E_{\mathrm{V}}$ relative to $E_{\mathrm{F}}$ in photoelectron 
DOS

(arb. units)
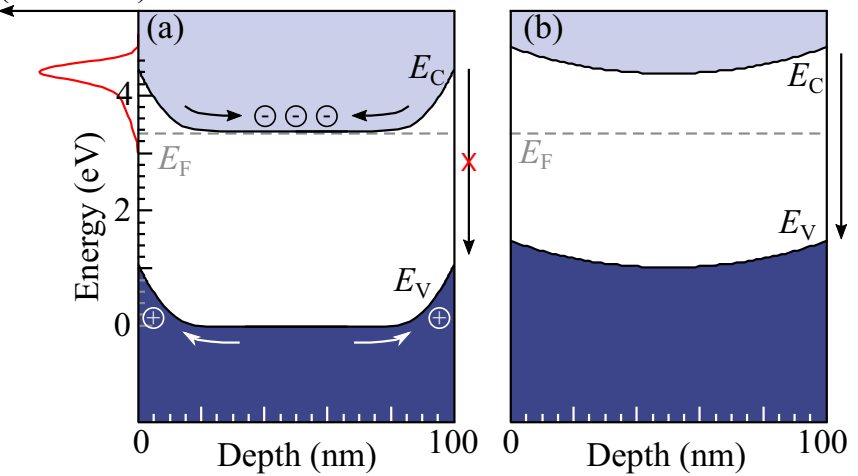

FIG. 4. Cross sections of the calculated energy bands in an $n$ type GaN NW with a diameter of $100 \mathrm{~nm}$ for a doping level of (a) $3 \times 10^{18} \mathrm{~cm}^{-3}$ and (b) $1 \times 10^{16} \mathrm{~cm}^{-3}$. The Fermi level is pinned by the surface state minimum, inducing an upward band bending at the side facets of the NW. The screening length of the band bending is shorter for higher doping levels and hence the charge separation of electron-hole pairs is more pronounced.

spectroscopy. Assuming a band gap of $\sim 3.4 \mathrm{eV}$, the energy of the surface state at the $\Gamma$ point is measured between $0.7 \mathrm{eV}$ [29] and $1.1 \pm 0.1 \mathrm{eV}$ [31] below $E_{\mathrm{C}}$. By fitting tunneling spectra, we found a similar value of $0.99 \pm 0.08 \mathrm{eV}$. As mentioned above, the onset voltage is determined by the Fermi-level pinning at the minimum of the empty Ga-derived surface state. Hence, our value agrees excellently with the surface state energies determined from Fermi-level pinning.

\section{Self-passivation of GaN nanowires}

Finally, the influence of surface states on GaN NWs is addressed. Figure 4 shows cross sections of the energy bands in an $n$-type GaN NW for different doping levels, calculated using a three-dimensional finite-difference approach $[39,40]$. In the calculations, we included the above-determined Ga-derived surface state minimum and electron affinity. The upward band bending toward the sidewall facets is due to the pinning of $E_{\mathrm{F}}$ at the surface state energy minimum. This shape of the band structure leads to a spatial separation of photo-generated electron-hole pairs, e.g., in solar cells, as indicated in Fig. 4(a). This spatial separation introduces a barrier (of $\sim 1 \mathrm{eV}$ ) for electron-hole pair recombination, reducing the recombination rate. This enhances the lifetime and efficiency of solar cells. However, it is detrimental for light-emitting devices. The Ga-derived surface state hence creates an electronic selfpassivation without additional deposited layers for solar cells. If the doping is lowered, the decay of the band bending is reduced, lifting the band edges upward in the center of the NW [see Fig. 4(b)]. Hence, the driving force for charge separation is lowered and the recombination probability is increased. Similarly, the conductivity is affected by a surface-pinninginduced band bending $[3,5]$.

\section{CONCLUSIONS}

In conclusion, for the nonpolar stoichiometric $\mathrm{GaN} m$-plane facet, we found an electron affinity of $4.06 \pm 0.07 \mathrm{eV}$, which is larger than most previous measurements performed on polar, usually nonstoichiometric surfaces. The minimum of the empty Ga-derived surface state is found $0.99 \pm 0.08 \mathrm{eV}$ below the conduction band minimum. This leads to an electronic self-passivation of GaN NWs due to an upward band bending, which spatially separates electrons and holes and hence increases the carrier lifetime. Thus, $n$-doped GaN NWs are a model system for surface-bulk charge separation, which can be expected to occur for all semiconductor NWs with surface states located in the band gap at the sidewall facets.

\section{ACKNOWLEDGEMENTS}

The authors thank R. Butté, J.-F. Carlin, and N. Grandjean, LASPE, Ecole Polytechnique Fédérale de Lausanne, Switzerland, for providing the samples and fruitful discussions as well as K.-H. Graf for technical support.
[1] K. A. Bertness, A. Roshko, L. M. Mansfield, T. E. Harvey, and N. A. Sanford, Nucleation conditions for catalyst-free GaN nanowires, J. Cryst. Growth 300, 94 (2007).

[2] L. Largeau, D. L. Dheeraj, M. Tchernycheva, G. E. Cirlin, and J. C. Harmand, Facet and in-plane crystallographic orientations of $\mathrm{GaN}$ nanowires grown on $\mathrm{Si}(111)$, Nanotechnology $\mathbf{1 9}$, 155704 (2008).

[3] R. Calarco, M. Marso, T. Richter, A. I. Aykanat, R. Meijers, A. v. d. Hart, T. Stoica, and H. Lüth, Size-dependent photoconductivity in MBE-grown GaN-nanowires, Nano Lett. 5, 981 (2005).

[4] Y. Dan, K. Seo, K. Takei, J. H. Meza, A. Javey, and K. B. Crozier, Dramatic reduction of surface recombination by in situ surface passivation of silicon nanowires, Nano Lett. 11, 2527 (2011).

[5] P. Capiod, T. Xu, J. P. Nys, M. Berthe, G. Patriarche, L. Lymperakis, J. Neugebauer, P. Caroff, R. E. Dunin-Borkowski, $\mathrm{Ph}$. Ebert, and B. Grandidier, Band offsets at zincblende-wurtzite GaAs nanowire sidewall surfaces, Appl. Phys. Lett. 103, 122104 (2013).
[6] A. Nussbaum, The theory of semiconducting junctions, Semicond. Semimet. 15, 39 (1981).

[7] J. S. Escher, NEA semiconductor photoemitters, Semicond. Semimet. 15, 195 (1981).

[8] J. I. Pankove and H. Schade, Photoemission from GaN, Appl. Phys. Lett. 25, 53 (1974).

[9] V. M. Bermudez, T. M. Jung, K. Doverspike, and A. E. Wickenden, The growth and properties of $\mathrm{Al}$ and $\mathrm{AlN}$ films on GaN(0001)-(1 × 1), J. Appl. Phys. 79, 110 (1996).

[10] V. M. Bermudez, Study of oxygen chemisorption on the $\mathrm{GaN}(0001)-(1 \times 1)$ surface, J. Appl. Phys. 80, 1190 (1996).

[11] C. I. Wu and A. Kahn, Investigation of the chemistry and electronic properties of metal/gallium nitride interfaces, J. Vac. Sci. Technol. B 16, 2218 (1998).

[12] C. I. Wu, A. Kahn, N. Taskar, D. Dorman, and D. Gallagher, GaN $(0001)-(1 \times 1)$ surface: Composition and electronic properties, J. Appl. Phys. 83, 4249 (1998). 
[13] S. P. Grabowski, M. Schneider, H. Nienhaus, W. Mönch, R. Dimitrov, O. Ambacher, and M. Stutzmann, Electron affinity of $\mathrm{Al}_{x} \mathrm{Ga}_{1-x} \mathrm{~N}(0001)$ surfaces, Appl. Phys. Lett. 78, 2503 (2001).

[14] K. M. Tracy, W. J. Mecouch, R. F. Davis, and R. J. Nemanich, Preparation and characterization of atomically clean, stoichiometric surfaces of $n$ - and $p$-type GaN(0001), J. Appl. Phys. 94, 3163 (2003).

[15] S.-C. Lin, C.-T. Kuo, X. Liu, L.-Y. Liang, C.-H. Cheng, C.-H. Lin, S.-J. Tang, L.-Y. Chang, C.-H. Chen, and S. Gwo, Experimental determination of electron affinities for $\mathrm{InN}$ and GaN polar surfaces, Appl. Phys. Express 5, 031003 (2012).

[16] P. Hacke, T. Detchprohm, K. Hiramatsu, and N. Sawaki, Schottky barrier on $n$-type GaN grown by hydride vapor phase epitaxy, Appl. Phys. Lett. 63, 2676 (1993).

[17] E. V. Kalinina, N. I. Kuznetsov, V. A. Dimitriev, K. G. Irvine, and C. H. Carter Jr., Schottky Barriers on $\mathrm{n}-\mathrm{GaN}$ grown on $\mathrm{SiC}$, J. Elec. Mat. 25, 831 (1996).

[18] R. Y. Korotkov, J. M. Gregie, and B. W. Wessels, Optical properties of the deep Mn acceptor in GaN:Mn, Appl. Phys. Lett. 80, 1731 (2002).

[19] P. G. Moses, M. Miao, Q. Yan, and C. G. Van de Walle, Hybrid functional investigations of band gaps and band alignments for AlN, GaN, InN, and InGaN, J. Chem. Phys. 134, 084703 (2011).

[20] Antoine Kahn, Fermi level, work function and vacuum level, Mater. Horiz. 3, 7 (2016).

[21] A. R. Smith, R. M. Feenstra, D. W. Greve, J. Neugebauer, and J. E. Northrup, Reconstructions of the GaN(0001) Surface, Phys. Rev. Lett. 79, 3934 (1997).

[22] J. E. Northrup and J. Neugebauer, Theory of GaN(1010) and (11̄̄0) surfaces, Phys. Rev. B 53, R10477 (1996).

[23] J. Wichert, R. Weber, L. Kipp, M. Skibowski, T. Strasser, F. Starrost, C. Solterbeck, W. Schattke, T. Suski, I. Grzegory, and S. Porowski, Angle resolved photoemission spectroscopy of gan (1010): Experiment and theory, Phys. Status Solidi B 215, 751 (1999).

[24] D. Segev and C. G. Van de Walle, Origins of Fermi-level pinning on $\mathrm{GaN}$ and $\mathrm{InN}$ polar and nonpolar surfaces, Europhys. Lett. 76, 305 (2006).

[25] C. G. Van de Walle and D. Segev, Microscopic origins of surface states on nitride surfaces, J. Appl. Phys. 101, 081704 (2007).

[26] L. Ivanova, S. Borisova, H. Eisele, M. Dähne, A. Laubsch, and $\mathrm{Ph}$. Ebert, Surface states and origin of the Fermi level pinning on nonpolar GaN(100) surfaces, Appl. Phys. Lett. 93, 192110 (2008).

[27] M. Bertelli, P. Löptien, M. Wenderoth, A. Rizzi, R. G. Ulbrich, M. C. Righi, A. Ferretti, L. Martin-Samos, C. M. Bertoni, and A. Catellani, Atomic and electronic structure of the nonpolar GaN(1100) surface, Phys. Rev. B 80, 115324 (2009).
[28] L. Lymperakis, P. H. Weidlich, H. Eisele, M. Schnedler, J.-P. Nys, B. Grandidier, D. Stievenard, R. E. Dunin-Borkowski, J. Neugebauer, and $\mathrm{Ph}$. Ebert, Hidden surface states at non-polar $\mathrm{GaN}(10 \overline{1} 0)$ facets: Intrinsic pinning of nanowires, Appl. Phys. Lett. 103, 152101 (2013).

[29] M. Himmerlich, A. Eisenhardt, S. Shokhovets, S. Krischok, J. Räthel, E. Speiser, M. D. Neumann, A. Navarro-Quezada, and N. Esser, Confirmation of intrinsic electron gap states at nonpolar $\mathrm{GaN}(1 \overline{1} 00)$ surfaces combining photoelectron and surface optical spectroscopy, Appl. Phys. Lett. 104, 171602 (2014).

[30] M. Landmann, E. Rauls, W. G. Schmidt, M. D. Neumann, E. Speiser, and N. Esser, GaN $m$-plane: Atomic structure, surface bands, and optical response, Phys. Rev. B 91, 035302 (2015).

[31] M. Franz, S. Appelfeller, H. Eisele, Ph. Ebert, and M. Dähne (unpublished).

[32] M. Schnedler, V. Portz, H. Eisele, R. E. Dunin-Borkowski, and $\mathrm{Ph}$. Ebert, Polarity-dependent pinning of a surface state, Phys. Rev. B 91, 205309 (2015).

[33] R. M. Feenstra, SEMITIP version 6, http://www.andrew. cmu.edu/user/feenstra/semitip_v6 (2011).

[34] J. Bono and R. H. Good, Theoretical discussion of the scanning tunneling microscope applied to a semiconductor surface, Surf. Sci. 175, 415 (1986).

[35] A. P. Wijnheijmer, J. K. Garleff, M. A. V. D. Heijden, and P. M. Koenraad, Influence of the tip work function on scanning tunneling microscopy and spectroscopy on zinc doped GaAs, J. Vac. Sci. Technol. B 28, 1086 (2010).

[36] R. M. Feenstra, J. A. Stroscio, J. Tersoff, and A. P. Fein, AtomSelective Imaging of the GaAs(110) Surface, Phys. Rev. Lett. 58, 1192 (1987).

[37] P. Lorenz, T. Haensel, R. Gutt, R. J. Koch, J. A. Schaefer, and S. Krischok, Analysis of polar GaN surfaces with photoelectron and high resolution electron energy loss spetroscopy, Phys. Status Sol. B 247, 1658 (2010).

[38] Y. Hinuma, A. Grüneis, G. Kresse, and F. Oba, Band alignment of semiconductors from density-functional theory and many-body perturbation theory, Phys. Rev. B 90, 155405 (2014).

[39] M. Schnedler, V. Portz, P. H. Weidlich, R. E. Dunin-Borkowski, and $\mathrm{Ph}$. Ebert, Quantitative description of photoexcited scanning tunneling spectroscopy and its application to the GaAs(110) surface, Phys. Rev. B 91, 235305 (2015).

[40] M. Schnedler, R. E. Dunin-Borkowski, and Ph. Ebert, Importance of quantum correction for the quantitative simulation of photoexcited scanning tunneling spectra of semiconductor surfaces, Phys. Rev. B 93, 195444 (2016). 